\title{
Prácticas educativas e interculturales como proceso formativo de jóvenes estudiantes de educación superior
}

\author{
Educational and intercultural practices as a training \\ process for young students of higher education
}

Emilia Cristina González-Machado ${ }^{1}$ Ernesto Israel Santillán-Anguiano ${ }^{2}$

\section{Resumen}

Se ha descrito la práctica educativa como experiencia comunitaria en un grupo de jóvenes estudiantes de la Universidad Autónoma de Baja California, institución de educación superior pública en el norte de México. Fue sustentada en el paradigma de la interculturalidad, para promover desde la Universidad, las dimensiones críticas y éticas como elementos de acercamiento a la diversidad social, cultural y económica en la comunidad indígena de Santa Catarina, ubicada en la región. La metodología fue cualitativa, desde una postura sociocrítica de la educación, por lo que supone no solo un nivel de profundización interpretativo, sino que busca incorporar transformaciones a las realidades sociales. Entre los principales resultados se identificaron cuatro aprendizajes generales expresados por los estudiantes participantes: 1) Conocimiento y aprendizaje a partir de experiencias, 2) Sensibilidad para ayudar y apoyar a la comunidad, 3) Las necesidades, historia, resistencia por los derechos y el territorio, y, 4) El diálogo como medio para eliminar la discriminación.

Como conclusiones se pone el acento en la posibilidad de generar aprendizajes significativos en la formación profesional universitaria a través de las experiencias interculturales que trasciendan el espacio áulico.

Palabras clave: Educación intercultural, enseñanza superior, estudiante universitario.

\section{Abstract}

The educational practice has been described as a community experience in a group of young students of the Autonomous University of Baja California, a public institution of higher education in northern Mexico. It was based on the paradigm of

\footnotetext{
1 Doctora en Ciencias de la Educación, profesora-investigadora de la Universidad Autónoma de Baja California. Correo: $\underline{\text { cristina. }}$ gonzalez@uabc.edu.mx, ORCID: https://orcid.org/o000-0002-9172-0956

2 Doctor en Ciencias y Humanidades para el Desarrollo Interdisciplinario, profesor-investigador de la Universidad Autónoma de Baja California. Correo: santillan_er@uabc.edu.mx, ORCID: https://orcid.org/oooo-0001-5954-8175
}

Recibido: 27/01/2021 - Aprobado: 10/11/2021 
interculturality to promote from the university, the critical and ethical dimensions as elements of approach to social, cultural and economic diversity in Santa Catarina indigenous community, located in the region. The methodology was qualitative from a socio-critical posture of education, which implies not only a level of interpretative deepening, but also seeks to incorporate transformations to social realities. Among the main results, four general learnings expressed by the participating students were identified: 1) Knowledge and learning from experiences, 2) Sensitivity to help and support the community, 3) Needs, history, resistance for rights and territory, and 4) Dialogue as a means to eliminate discrimination.

As conclusions, emphasis is placed on the possibility of generating significant learning in university professional training through intercultural experiences that transcend the classroom space.

Keywords: Intercultural education; higher education; university student.

\section{Introducción}

Cuando se vislumbra una propuesta educativa intercultural, esta debe estar basada en la práctica, pues el hecho de enunciar como intercultural una acción no la convierte por sí misma en un acto intercultural. Por el contrario, un modelo de educación que se asuma como intercultural requiere forzosamente transformar el discurso en acción (Gómez, 2018). Las instituciones educativas deben valorar el aporte de las culturas indígenas como formas posibles de aprendizajes, retomando las experiencias como modelos para la construcción de sociedades democráticas. En ese sentido la educación intercultural, tiene razones válidas para hacer aportaciones significativas (Morales et al., 2018).

En México la aproximación intercultural se encuentra aún incipiente, diversa y en condición desigual en distintos campos. Incluso en el ámbito de lo académico, las políticas públicas y por consiguiente los procesos de planeación educativa. Todavía es posible encontrar una postura tradicional detrás de los discursos que se asumen interculturales. No es de extrañar que las propuestas que se encuentren en la realidad mexicana, formen parte de un continuo heterogéneo y diverso, aun en el caso de las instituciones que se asumen como interculturales (Olivera y Dietz, 2017). Sin embargo, la reivindicación de posiciones que apuestan por el respeto a la diferencia y los derechos de los pueblos originarios se abre cada día paso para encontrar formas de trabajo que intentan ser novedosas (Rojas-Cortés et al., 2016).

La propuesta que se presenta, es un replanteamiento educativo que busca conceptualizar a la interculturalidad como un desarrollo y un plan de trabajo, donde sean retomadas otras formas de saber y construir la realidad dentro de espacios educativos a nivel superior. El objetivo es establecer vínculos con manifestaciones culturales 


\section{EDUCACIÓN}

distintas a las que los estudiantes se encuentran acostumbrados y predispuestos, para trascender a través del contacto con el otro las prácticas y el pensamiento tradicional basado en la diferencia, la exclusión y el racismo, los cuales no son asumidos en la práctica educativa, pues se encuentran encubiertos en un discurso supuestamente integrador.

En ese sentido, esta práctica pedagógica asume la interculturalidad en términos más amplios. Como un proyecto político, social, epistémico y ético (Collado, 2017) que busque la modificación de las condiciones que han generado históricamente un saber colonizador que es perpetuado hasta la actualidad y en el cual se requiere un debate que involucre a la sociedad en su conjunto. Es necesario, por lo tanto, que las prácticas educativas interculturales tengan como base la promoción de los derechos humanos, así como la generación y el respeto por la creación de formas distintas de acceder y construir conocimiento (Walsh, 2017).

\section{Revisión de literatura}

Nunca como antes en la historia de la humanidad, ha sido tan necesario el diálogo y la tolerancia como complemento de las relaciones sociales. Las condiciones de reestructuración económica que se han desarrollado a escala planetaria desde la década de los ochenta han desencadenado una serie de cambios en el orden político y social:

Igual que en el siglo XIX los seres humanos tuvieron que aprender... a someterse a las condiciones de la sociedad industrial y del trabajo asalariado, hoy y en el futuro tienen que aprender... a sentarse a una mesa y, más allá de las fronteras, encontrar e imponer soluciones para las amenazas que ellos mismos han causado. (Beck, 2008, p.54)

En ese sentido, la cultura como manifestación de los fenómenos sociales no ha sido la excepción. Los movimientos migratorios son muestra de la dinámica diversa y de una definición constante de las fronteras territoriales, las fluctuaciones económicas y las crisis políticas permanentes. La sociedad ha transitado de una multiplicidad de comunidades asentadas en el campo, cuyas características son precisamente las culturas tradicionales, arraigadas, uniformes y con robustas raíces indígenas, a un conglomerado de comunidades urbanas, heterogéneas con interacciones mediadas por las tecnologías de la comunicación.

Ahora bien, si un grupo social puede definirse a partir de la relación entre los individuos que lo integran y la forma en cómo estos se distinguen a su vez de otros grupos sociales; la identidad del grupo se basa en la capacidad que tiene el colectivo para asumir como propias las manifestaciones simbólicas y culturales con las cuales se identifica (Santillán, 2018). 
Es en estos escenarios que se desarrolla el concepto de interculturalidad. La UNESCO la define como la construcción de relaciones equitativas entre individuos, comunidades, culturas y países. En el ámbito educativo, la interculturalidad asume la existencia de diálogo entre iguales en condiciones de equidad. Reconoce la importancia de los saberes locales y las culturas como uno de los aportes más importantes en la educación contemporánea (Organización de la Naciones Unidas para la Educación, la Ciencia y la Cultura, 2017).

La categoría se convierte en el eje discursivo educativo, incorpora la capacidad de individuos o grupos para comunicarse entre ellos a pesar de provenir de distintas culturas. La interculturalidad responde a elementos de igualdad, respeto y sentido de democracia que cuestionen y limiten las relaciones de dominación (Schmelkes, 2021). De esta forma puede entenderse que la interculturalidad promueve de forma activa el acercamiento entre identidades y culturas en distintos espacios sociales, en el caso de la educación, se adscribe a la aplicación de las prácticas educativas. Contextualmente, intenta establecer puentes de comunicación entre distintas visiones del mundo desde una perspectiva equitativa más que igualitaria (Navarro y Saldívar, 2020).

Existen peligros inherentes al uso indiscriminado del concepto de interculturalidad. Siglos de dominación sobre las culturas subalternas se convierten en muchos casos en demagogia o discurso como acto misericordioso. La cultura occidental difícilmente se presta a comunicarse de manera igualitaria con culturas distintas. Cualquier propuesta o política que intente ser intercultural, requiere eliminar la tentación de imponer su punto de vista (Olivar, 2020).

El discurso predominante, sigue alimentado de una tradición epistémica positivista a pesar de existir nuevos discursos en el campo educativo. Se mantiene la visión universalista como propuesta pedagógica en la era moderna, cuyo fin último apuesta a una homogeneización de la humanidad. Este discurso clasifica y categoriza a los individuos de cada grupo, incluyendo sus necesidades educativas y culturales (Pallarès y Chiva, 2018).

Para Maldonado y del Valle (2016) es posible conceptualizar la interculturalidad a partir de diferentes puntos de vista. En primer lugar, se asume como un fenómeno comunicativo que se desarrolla entre individuos que se asumen desde culturas distintas. Por lo tanto, su uso queda circunscrito a una actividad discursiva y simbólica. Esta primera aproximación se encuentra contextualizada a partir de los fenómenos de movilidad y desterritorialización que se han manifestado en el siglo XXI, así como la aparición de los medios tecnológicos que posibilitan la comunicación a escala global.

La segunda aproximación, tiende a ver a la interculturalidad desde una perspectiva política. Su reconocimiento es otorgado a partir de un marco normativo internacional basado en los derechos humanos y que tiene su contraparte en legislaciones nacionales 


\section{EDUCACIÓN}

con posiciones colonialistas respecto a su relación con los pueblos originarios. Esta segunda postura asume la crítica al Estado nación como un mito que genera un discurso de legitimación de lo diferente, pero que en la realidad reproduce una estructura de segregación y exclusión de lo diferente.

La tercera forma de acercarse a la interculturalidad es desde una visión interdisciplinaria. Desde la óptica de la academia, la interculturalidad ha sustituido en muchos aspectos a la categoría de identidad como concepto esencialista. En ese sentido los principales aportes teóricos giran en torno al análisis del entramado histórico-cultural que abarca grandes poblaciones. En especial en Latinoamérica la visión ha desarrollado su principal aporte respecto a los estudios decoloniales y su huella en las relaciones sociales en la era moderna.

El cuarto sentido al concepto, ha surgido a partir de los movimientos sociales. Esta última etapa de aproximación, la concibe como proyecto político en construcción como una forma de acercarse a la propia identidad, así ha sido manifestado por los principales movimientos indígenas y afrocaribeños en la región latinoamericana. Concebir la interculturalidad desde una visión subalterna, ha permitido visibilizar a la diversidad de actores y subjetividades que han pasado desapercibidas en otros momentos (Maldonado y del Valle, 2016).

El concepto de interculturalidad debe fungir como una herramienta descriptiva y analítica. Su definición implica al conglomerado de interrelaciones que estructuran una sociedad en términos culturales, étnicos, lingüísticos, o de identidad. En otras palabras, la interculturalidad es un ensamblaje que organiza las relaciones entre grupos diferenciados, los cuales se relacionan a partir de una dinámica de mayoríaminoría. Este tipo de relaciones asiduamente son de carácter asimétrico y revelan las formas en que históricamente se visualiza o se esconde la diversidad. En el caso de la discriminación, esta es legitimada a partir de identificarla con grupos particulares de una sociedad (Dietz, 2017).

En el mismo sentido, el concepto ha prevalecido en Europa lo que ha mantenido el debate en torno a la diversidad cultural al interior de los organismos supranacionales como en el caso de la UNESCO y la Unión Europea. Por otro lado, el concepto ha sido reivindicado de manera distinta en Latinoamérica por los movimientos indígenas, para posicionar las resistencias de carácter descolonizador.

La interculturalidad juega un papel importante cuando logra desvincularse precisamente del discurso hegemónico colonial, que intenta orientar el debate hacia ideas asociadas al progreso, la modernidad y la salvación de los pueblos originarios (Aman, 2014). La interculturalidad busca y asume, por el contrario, otras formas de conocimiento, subjetividad y visiones sobre el mundo. 
Estermann (2018) apunta en torno al papel colonizador de los llamados proyectos de modernización, democratización e incorporación al mercado global que se encuentra dentro del discurso "incluyente". Dicho discurso es en apariencia emancipador y promotor de la interculturalidad, sin embargo, asume una actitud patriarcal y asistencial que encubre una propuesta de dominación. En dicha propuesta, existe claramente el papel de un sujeto activamente "incluyente", mientras que del otro lado sobrevive un objeto pasivo que requiere ser "incluido". El discurso basado en la modernidad, la tecnología, la participación y el consumo es el discurso disfrazado del colonialismo.

De hecho, la llamada interculturalidad crítica (Ortega-Caicedo et al., 2020) no se formula ya como una dicotomía entre diversidad/diferencia; por el contrario, centra su análisis en una visión sobre la diferenciación, la cual es construida dentro de una estructura colonial marcadamente jerárquica y racial, donde los blancos -y después los mestizos-, se encuentran por encima de los pueblos indígenas y afrodescendientes. A partir de ahí se construye una perspectiva que es asumida desde la subalternidad, que requiere la transformación de las estructuras para generar condiciones de existencia distintas. El punto de partida para una mirada intercultural en Latinoamérica nace del reconocimiento de las llamadas culturas periféricas (Dussel, 2016).

El supuesto diálogo entre culturas asume que todas ellas parten de un sistema de relaciones simétricas. De hecho, el concepto mismo de Estado multicultural, encierra la manifestación de la visión occidental institucionalizada y severamente agresiva con las culturas subalternizadas.

En la actualidad, desde un punto de vista que intente enfrentarse a cualquier manifestación de dominación o explotación, debe esforzarse por eliminar lo que se denomina colonialidad del poder (Soto, 2019). Esto es, evidenciar el papel del patrón universal de explotación inherente a las visiones eurocéntricas que siguen avivando las manifestaciones de explotación y dominación tanto materiales como culturales.

En México, los índices más altos de rezago económico los presentan las comunidades con población mayoritariamente indígena. La evidencia demuestra la relación directa entre pertenecer a una comunidad indígena y ser pobre. Para la Comisión Económica para América Latina y el Caribe, en los años que comprenden el periodo 2003-2017, la desigualdad en México se ha incrementado (Puyana, 2015).

La disparidad entre el ritmo de aumento del producto y el de la riqueza provoca una mayor distinción entre ricos y pobres (del Castillo, 2017). La tasa de pobreza extrema de la población indígena en México es cuatro veces mayor que la del resto de la población. Un $38 \%$ de la población indígena vive en pobreza extrema, en comparación con un 10\% de la población en general (Esquivel, 2015). 
De acuerdo a la Encuesta Intercensal 2015 (Instituto Nacional de Estadística y Geografía, 2016), casi la mitad (46.9\%) de la población indígena en México, mayor de 15 años, es económicamente activa. Un 15\% de la población trabajadora indígena no recibe salario alguno por su trabajo, en comparación con un $2 \%$ de la población en general. En el caso particular de las mujeres, un 32.2\% son trabajadoras por su cuenta, en contraste con un $19 \%$ de las mujeres no indígenas.

La vulnerabilidad en salud se define como la falta de protección de grupos de población frente a riesgos potenciales por recursos nulos o limitados, ya sean personales, familiares, sociales, económicos o institucionales. Casi el 8o\% de la población indígena en México subsiste en una situación de pobreza o pobreza extrema. De estos, un $20 \%$ trabaja en condiciones de salarios con baja remuneración y un $21 \%$, ni siquiera recibe pago por su trabajo. Cerca del 90\% de la población entre 6 y 14 años de edad se dedica a actividades agrícolas o ganaderas. En las comunidades indígenas es común la mortalidad infantil, las muertes maternas, las enfermedades crónicas y la desnutrición. Más del 80\% de la población indígena no cuenta con seguridad social (Juárez et al., 2014).

\section{Materiales y métodos}

Entre los años 2010 a 2019, un grupo de profesores y profesoras de la Universidad Autónoma de Baja California, establecimos comunicación con mujeres artesanas de la comunidad de Santa Catarina. El objetivo central era acompañarlas en un proceso de diseño de un proyecto ecoturístico, que finalmente no pudo concretarse por razones externas a la comunidad, por lo que se desistió de él a finales del 2014. A lo largo de estos años la comunicación se ha mantenido constante, aunque esporádica, tratando de continuar los lazos y vínculos con la comunidad. Desde el año 2015 hasta el 2019, se planificaron una serie de acciones para que un mayor número de profesores, profesoras y estudiantes pudieran compartir la experiencia con la comunidad, y al mismo tiempo apoyar en proyectos específicos de interés común.

El acercamiento a la realidad de las comunidades indígenas, permite la construcción de un conocimiento colectivo. Las visitas a la comunidad de Santa Catarina fueron organizadas como opciones de aprendizaje. Para el grupo de participantes era vital que se pudieran adquirir vivencias formativas (Deroncele et al., 2020), al mismo tiempo que se desarrollaban los contenidos de las asignaturas.

Se intentó trastocar el imaginario que asocia al conocimiento como producto del esfuerzo individual, en un espacio institucionalizado y trascender hacia un aprendizaje colectivo en contacto con problemáticas reales. El llamado aprendizaje situado (Álvarez, 2020), tiene una larga tradición en proponer el desarrollo de habilidades y conocimientos disciplinarios a partir de la solución de problemas sociales o comunitarios. Esto cobra especial relevancia cuando se trabaja en escenarios donde históricamente 
ha existido una relación de violencia, despojo, pobreza y vulnerabilidad como sucede con las comunidades indígenas del continente.

La propuesta de trabajo busca incidir en el trayecto formativo y profesional de estudiantes como actores dentro de una dinámica de relaciones, donde estos interactúan con un grupo diverso de personas a partir de distintas necesidades. En ese sentido, el objetivo principal busca afianzar la interacción de participantes, con la idea de que los procesos de formación del conocimiento se realizan a través de dinámicas sociales.

La formación profesional se conforma a partir de elementos culturales, sociales, políticos y ecológicos (Cuadra-Martínez et al., 2018), los cuales difícilmente pueden adquirirse desde la óptica de una disciplina aislada o a partir de prácticas de aprendizaje desvinculadas del contexto de las necesidades sociales. Para tal efecto, se busca considerar tres elementos:

a) La dinámica de los grupos: Las estrategias didácticas buscan enlazar la práctica educativa con la experiencia de vida de integrantes de la comunidad. Este proceso genera un espacio socioafectivo que favorece el pensamiento reflexivo y creativo (Roque et al., 2018). El grupo de estudiantes intenta cumplir con las metas establecidas, pues existe el compromiso con las personas participantes de la comunidad para alcanzar los objetivos. En otras palabras, el desarrollo de las metas académicas se enlaza con el cumplimiento de los objetivos del trabajo cooperativo.

b) Equipo facilitador: Ahora bien, durante este proceso es importante el rol de la planta docente como facilitadores (Quevedo y Arruti, 2018). Al buscar intervenir para promover la interacción y el diálogo, a partir de identificar al grupo y la comunidad como facilitadores, buscan activar el encuentro de estudiantes e integrantes de la comunidad sobre objetivos comunes, promoviendo la tolerancia, el respeto, el diálogo, como base del trabajo en conjunto.

c) El espacio físico: Uno de los componentes que hace más atractiva la propuesta, es el cambio de la experiencia situada en la comunidad (Pérez, 2017) en comparación con el aprendizaje realizado en aulas tradicionales.

La actividad por sí misma intenta romper con la rigidez de los espacios institucionales. La propuesta asume, que otra forma de promover los derechos humanos y los procesos democráticos en la dinámica de aprendizaje se construyen por experiencias alternativas y promuevan el diálogo, así como la participación.

La idea central que envuelve a este trabajo, es vincular la labor de docentes y estudiantes a partir de un contacto directo con una comunidad con necesidades sociales específicas. La propuesta se enfoca en aprendizajes y experiencias que promuevan el pensamiento crítico y el compromiso ético, articulando el conocimiento y 
los fenómenos locales. En este sentido, se busca trascender la visión de la institución escolar que mantiene una afinidad directa con el pensamiento europeo, con atención especial a la promoción de la alta cultura y la modernidad. Este proyecto hace hincapié en un acercamiento a una visión más heterogénea y con un fuerte sentido intercultural de la educación.

\section{Resultados y discusión}

El grupo de participantes se compuso por 30 jóvenes estudiantes con adscripción en los programas de las licenciaturas de Sociología, Educación y Psicología en la Facultad de Ciencias Humanas de la Universidad Autónoma de Baja California, una institución de educación superior pública en el noroeste de México. En el grupo hubo una mayor proporción de mujeres (26) que de hombres (4), con una edad promedio de 25 años.

El instrumento que se utilizó para este reporte se refiere a una guía de entrevista semiestructurada, que se respondió de manera individual a partir de las notas de campo, está compuesta de seis preguntas abiertas que exploran las siguientes dimensiones desde la perspectiva del grupo de participantes: 1) La descripción general sobre la comunidad nativa; 2) Las necesidades que observaron en la comunidad; 3) Los aprendizajes obtenidos en la práctica educativa.

A partir de la descripción general de la comunidad indígena por parte del grupo de estudiantes participantes, se pudieron identificar tres elementos: 1) La percepción de la comunidad en el sentido de sus características, 2) La comunidad en cuanto a la población y, 3) La organización de la población. La descripción de los participantes giró en torno a caracterizar a la comunidad como pequeña, sencilla, cordial, cálida. Con un sentido de organización social tendiente a la hospitalidad, la sabiduría, la hermandad. Amigable y abierta al exterior; trabajadora, cálida y servicial al interior (ver figura 1). 


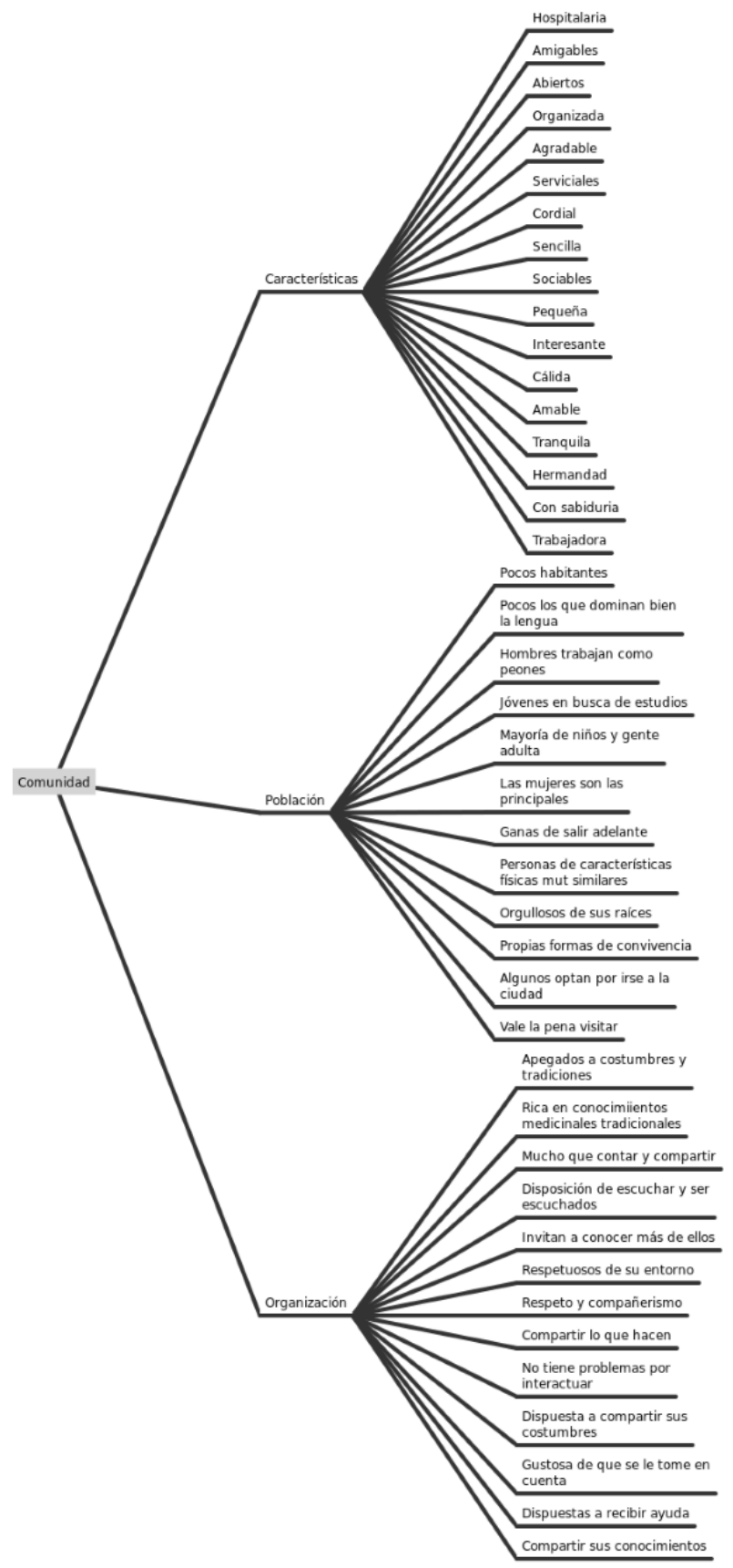

Figura 1: Descripción de la comunidad a partir de la percepción de estudiantes.

Fuente: elaboración propia. 


\section{EDUCACIÓN}

La comunidad se caracterizó como pequeña y con un fuerte sentido de tradición, apego a su cultura y creencias. La cultura fue percibida como rica, especialmente en lo referente al uso de la lengua original, donde existe una pérdida de la misma por las generaciones más jóvenes. Del mismo modo, la manifestación material cultural más significativa fue la elaboración de artesanía, en especial aquella que hace uso respetuoso de los elementos medioambientales como es el caso de la cestería, cerámica y la bisutería.

En relación con los elementos inmateriales, son los cantos y los bailes tradicionales los que fueron identificados como esenciales para el grupo de jóvenes estudiantes. $\mathrm{Al}$ ser Santa Catarina una comunidad indígena con niveles elevados de pobreza y marginación, era importante para el proyecto identificar la forma en que estos elementos eran percibidos por el grupo de estudiantes.

Fue recurrente la descripción de una comunidad humilde, alejada de los centros urbanos, con recursos limitados y escasos servicios. Sin embargo, esta descripción contrasta con una percepción influenciada por el contacto directo con los pobladores a quienes observan como personas con intenciones de mejorar económicamente, donde se entiende y se justifica la migración a la ciudad como parte de la búsqueda de mejores condiciones de vida, con una necesidad de la población joven para buscar oportunidades educativas. Se percibe a las mujeres como las principales promotoras de la economía familiar, al desarrollar proyectos artesanales como alternativas al trabajo esporádico. El abandono de los servicios básicos por parte del Estado y sus políticas públicas, fue una constante que refuerza la idea de marginación y pobreza.

En relación con la pregunta que explora las necesidades observadas por el grupo de estudiantes en la comunidad, las respuestas se pueden agrupar en cuatro categorías. A) La primera, que hace referencia a la cultura, en la que se puede apreciar la necesidad de rescatar las costumbres del grupo étnico, así como la lengua nativa. B) La segunda, se refiere a la necesidad de contar con oportunidades educativas para la población joven, así como la capacitación en distintos oficios para las mujeres de la comunidad. C) La tercera, que identifica requerimientos de atención médica, proveer medicamentos a la población, así como estrategias de prevención de enfermedades como la diabetes y las adicciones. D) La última, la que mayormente se enunció por el grupo de estudiantes, se relaciona con la economía y los servicios, tiene que ver con aspectos como la falta de fuentes de empleo, de transporte para trasladarse a conseguir recursos para satisfacer la canasta básica, necesidades de abastecimiento de agua y despensa (ver figura 2). 


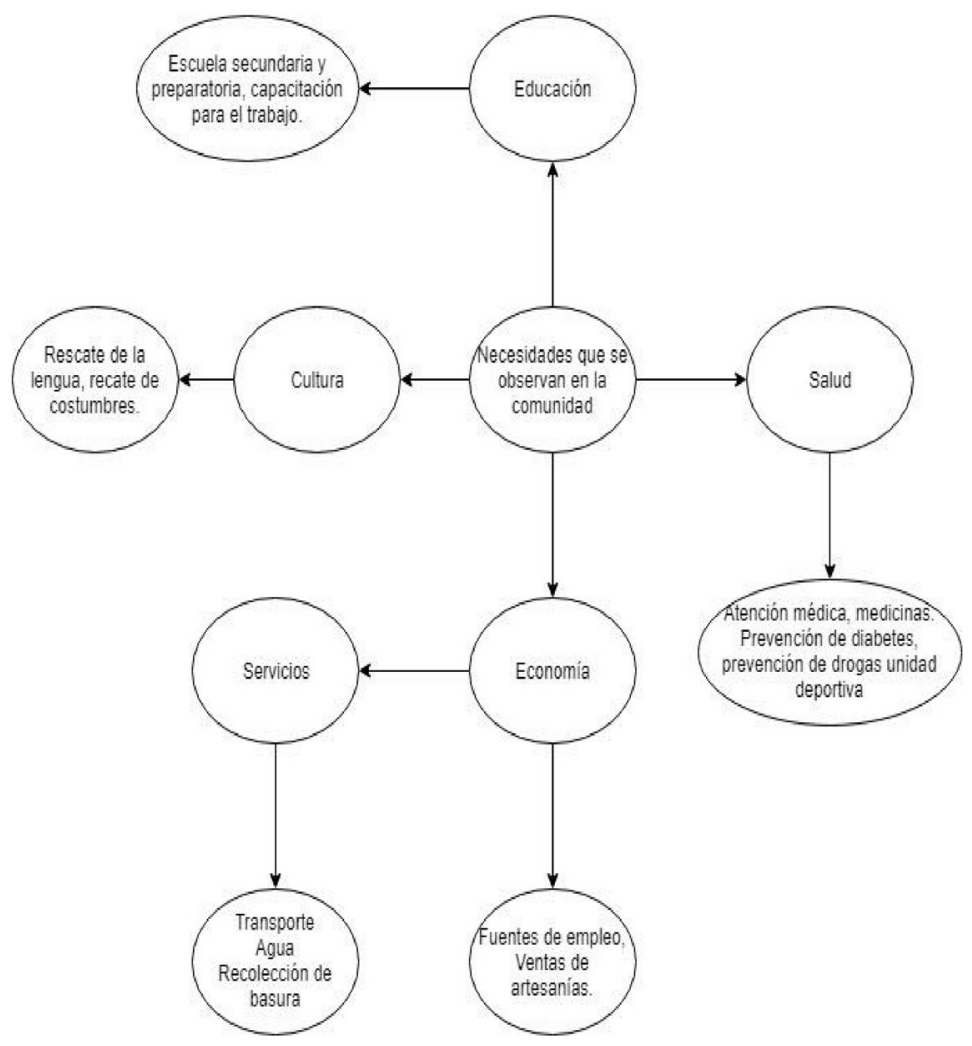

Figura 2: Esquema de las necesidades de la comunidad observadas por estudiantes.

Fuente: elaboración propia

En cuanto a los resultados de la experiencia de la práctica educativa, se puede observar en la tabla 1, que estos se agrupan en cuatro aprendizajes significativos:

Tabla 1: Aprendizajes de estudiantes

\begin{tabular}{|l|l|}
\hline \multicolumn{1}{|c|}{ Aprendizaje } & \multicolumn{1}{|c|}{ Lo expresado por estudiantes } \\
\hline $\begin{array}{l}\text { Capacidad de aplicar los conoci- } \\
\text { mientos en la práctica }\end{array}$ & Conocimiento y aprendizaje a partir de experiencias \\
\hline $\begin{array}{l}\text { Responsabilidad social y compromi- } \\
\text { so ciudadano }\end{array}$ & $\begin{array}{l}\text { Sensibilidad para ayudar y apoyar a la comunidad con } \\
\text { respeto a sus necesidades, historia, cultura, creencias y } \\
\text { tradiciones }\end{array}$ \\
\hline $\begin{array}{l}\text { Compromiso con el medio socio- } \\
\text { cultural }\end{array}$ & $\begin{array}{l}\text { Reconocer a los otros, los esfuerzos que hacen para } \\
\text { vivir, los procesos de resistencia y lucha por derechos } \\
\text { y recursos. }\end{array}$ \\
\hline Compromiso ético & Diálogo, eliminar la discriminación \\
\hline
\end{tabular}




\section{EDUCACIÓN}

En cuanto a la capacidad para aplicar los conocimientos en la práctica, dentro de las respuestas, el grupo de estudiantes hace referencia a la oportunidad de aprender en/con la comunidad, desde aspectos de derecho, historia, educación de los pueblos originarios en México:

Pude notar las diferencias que existen, así como las similitudes, por ejemplo, que siempre a las comunidades se les deja al último, al estar dentro de la urbanidad olvidamos estas pequeñas poblaciones que forman parte de la historia. La práctica en esta comunidad fue una de las mejores experiencias. (Estudiante de Educación, 22 años, 19 de mayo de 2017)

En relación con la responsabilidad social y compromiso ciudadano, es relevante la respuesta de una estudiante:

Que al participar o intervenir en este y muchos contextos, es necesario poner a un lado lo que nosotros asumimos como necesidades en papel de "expertos", ya que serán las personas con las que trabajaremos las verdaderas expertas en su situación. (Estudiante de Psicología, 21 años, 12 de mayo de 2017)

En cuanto al compromiso con el medio sociocultural, se identifica la respuesta de otra estudiante:

Fue muy interesante para mí conocer la manera en que han luchado desde años atrás por obtener sus derechos y recursos, el saber que muchas personas los han discriminado siendo muchas veces los mismos mexicanos, es triste ya que son parte de nosotros. (Estudiante de Psicología, 21 años, 20 de mayo de 2017)

El compromiso ético se pone de manifiesto en la narrativa de una estudiante al mencionar:

Sin duda alguna, es una experiencia que personalmente me encantó, poder convivir con personas llenas de ganas de compartir su historia con nosotros, que quieren dar a conocer más de ellos, de sus tradiciones y costumbres, es algo que no se vive tan a menudo en la sociedad en la que nos encontramos y es algo diferente que experimentar y que deja un enriquecimiento enorme. (Estudiante de Psicología, 22 años, 25 de mayo de 2017)

\section{Conclusiones}

Una perspectiva educativa que tenga sus fundamentos en la diversidad, no se desarrolla exclusivamente en el terreno de lo abstracto, requiere reconocer la diversidad social existente en el mundo. Necesita el acercamiento real a lo diverso y lo diferente, que rompa los estereotipos existentes en la cultura mestiza, y que ayude a erradicar 
el racismo en la educación mexicana (Velasco y Baronnet, 2016). Se intenta acercar a todo el grupo de participantes a la realidad indígena regional y facilitar una visión que integre la concepción de lo diverso como elemento articulador del contacto entre culturas (Ruiz-Bernardo, 2018).

Dentro de los grandes retos que la educación del siglo XXI enfrenta, es la interculturalidad como un elemento indisociable de una sociedad globalizada, donde aparentemente las fronteras se disuelven. La interculturalidad entendida como una expresión de las realidades comunitarias subalternas, y no solo como un discurso educativo que se asume como intercultural pero que obliga al estudiante a un aprendizaje tangencial, y carente de significación. No es solo una forma distinta de abordar el acercamiento cultural, donde es común que se desarrolle un discurso integrador, colonial e incapaz de asumir un carácter crítico hacia las prácticas educativas que perpetúan el racismo y la discriminación en forma de políticas educativas.

Finalmente, la experiencia educativa desde una perspectiva intercultural crítica, resalta un proceso que logra:

1- Articular la teoría y la práctica para la solución de problemas complejos en espacios reales.

2- Contextualizar realidades socioculturales diversas que interpelan la postura ontológica, epistemológica y política, lo que se traduce en la forma de manifestar un compromiso ético de forma crítica.

3- Revalorar los conocimientos en la práctica fuera del aula para desarrollar aprendizajes significativos en la formación profesional.

4- Promover experiencias basadas en actividades solidarias, que implican diálogo y la interacción con otras realidades sociales, lo que posibilita el acercamiento con los otros.

\section{Lista de referencias}

Álvarez, M. (2020). Aprendizaje situado: mito o realidad desde el aprendizaje basado en problemas. Revista Espacios, 41(28), 327-337. https://www.revistaespacios. com/a2ov41n28/a2ov41n28p26.pdf

Aman, R. (2014). Why interculturalidad is not Interculturality: Colonial remains and paradoxes in translation between indigenous social movements and supranational bodies. Cultural Studies, (2), 1-24. https://doi.org/10.1080/0950238 6.2014 .899379

Beck, U. (2008). La sociedad del riesgo mundial. Barcelona: Paidos. 


\section{EDUCACIÓN}

Collado, J. (2017). Interculturalidad y descolonialidad: Retos y desafíos epistemológicos, Revista nuestrAmérica; 5 (9), 37-57. http://repositorio.unae.edu.ec/ bitstream/5600o/96/1/Texto.pdf

Cuadra-Martínez, D., Castro, P., y Juliá, M. (2018). Tres Saberes en la Formación Profesional por Competencias: Integración de Teorías Subjetivas, Profesionales y Científicas. Formación universitaria, 11(5), 19-30. https://dx.doi.org/10.4067/ So718-50062018000500019

Del Castillo, M. (2017). La distribución y desigualdad de los activos financieros y no financieros en México. México: CEPAL.

Deroncele, A., Medina, P., y Gross, R. (2020). Gestión de potencialidades formativas en la persona: reflexión epistémica y pautas metodológicas. Revista Universidad y Sociedad, 12(1), 97-104. http://scielo.sld.cu/scielo. php?script=sci_arttext\&pid=S2218-36202020000100097\&lng=es\&tlng=es

Dietz, G. (2017). Interculturalidad: una aproximación antropológica. Perfiles Educativos, (156), 192-207. https://www.redalyc.org/articulo.oa?id=13250923012

Dussel, E. (2016). Transmodernidad e interculturalidad. Astrágalo, (21), 31-54. https:// dialnet.unirioja.es/servlet/articulo?codigo $=7008889$

Esquivel, G. (2015). Desigualdad extrema en México. Concentración del poder económico y político. México: Oxfam.

Estermann, J. (2018). Interculturalidad y conocimiento andino: reflexiones acerca de la monocultura Epistemológica. Revista Kawsaypacha: Sociedad Y Medio Ambiente, (2), 11-32. https://doi.org/10.1880o/kawsaypacha.201801.001

Gómez, M. (2018). Competencias interculturales. Estudio sobre competencias interculturales en instructores comunitarios de la modalidad de atención educativa en el estado de Chiapas. Monterrey: Nómada.

Instituto Nacional de Estadística y Geografía. (2016). Encuesta Intercensal 2015. México: Instituto Nacional de Estadística y Geografía. https://www.inegi.org. $\mathrm{mx} /$ programas/intercensal/2015/

Juárez-Ramírez, C., Márquez-Serrano M., Salgado de Snyder N., Pelcastre-Villafuerte B., Ruelas-González, M., Reyes-Morales, H. (2014). La desigualdad en salud de grupos vulnerables de México: adultos mayores, indígenas y migrantes. Revista Panamericana de Salud Pública; (4), 284-29o. https://scielosp.org/pdf/ rpsp/2014.v35n4/284-29o/es 
Maldonado, C. y del Valle, C. (2016). Episteme decolonial en dos obras del pensamiento Mapuche: Re-escribiendo la interculturalidad. Chungará, 48 (2), 319-329. http:// dx.doi.org/10.4067/So717-73562016005000018

Morales, S., Quintriqueo, S. Uribe, Pilar, A., y Arias, K. (2018). Interculturalidad en educación superior: experiencia en educación inicial en La Araucanía, Chile. Convergencia, 25 (77), 55-76. https://doi.org/10.29101/crcs.v25i77.4706

Navarro, S. y Saldívar, A. (2020). El papel de la interculturalidad en la construcción de sujetos identitarios: La experiencia de la Universidad Intercultural de Chiapas en el municipio de Oxchuc (UNICH-Oxchuc). DIDAC, (76), 24-31. https://doi. org/10.48102/didac.2020.76 JUL-DIC.24

Olivar, A. (2020). Políticas públicas y enfoques diferenciales: aproximaciones desde la interculturalidad y la democracia radical. Iztapalapa. Revista de ciencias sociales y humanidades, 41 (88), 139-162.https://doi.org/10.28928/ri/882020/ aot1/olivarrojasa

Olivera, I. y Dietz, G. (2017). Educación superior y pueblos indígenas: marcos nacionales para contextualizar. Anthropologica, 35 (39), 5-23. https://dx.doi.org/10.1880o/ anthropologica.201702.001

Organización de la Naciones Unidas para la Educación, la Ciencia y la Cultura. (2017). Competencias interculturales. Marco conceptual y operativo. Bogotá: Organización para la Educación, la Ciencias y la Cultura, Universidad Nacional de Colombia.

Ortega-Caicedo, A. y Lang, M. (2020). Gritos, grietas y siembras de nuestros territorios del sur. Quito: Abya-Yala.

Pallarès, P. y Chiva, O. (2018). El lugar del individuo en la era post-postmoderna. Sociedad, educación y ciudadanía tras la postmodernidad. Pensamiento. Revista De Investigación E Información Filosófica, 74 (282), 835-852. https://doi. org/10.14422/pen.v74.i282.y2018.004

Pérez, G. (2017). El aprendizaje situado ante una teoría constructivista en la posmodernidad. Glosa, 5 (8), 1-14. https://static1.squarespace.com/ static/53b1eff6e4boe8agf63530d6/t/5a55564e652dea613b15c15o/1515542096177/ Articulo+aprendizaje+situado.pdf

Puyana, A. (2015). Desigualdad horizontal y discriminación étnica en cuatro países latinoamericanos. Notas analíticas para una propuesta política. México: CEPAL. 


\section{EDUCACIÓN}

Quevedo, E. y Arruti, A. (2018). El nuevo rol del docente como facilitador del aprendizaje, en A. Villa (Ed.). Tendencias actuales de las transformaciones de las universidades en una nueva sociedad digital. Madrid: Foro Internacional de Innovación Universitaria.

Rojas-Cortés, A. y González-Apodaca, E. (2016). El carácter interactoral en la educación superior con enfoque intercultural en México. LiminaR, 14(1), 73-91. http://www. scielo.org.mx/scielo.php?script=sci_arttext\&pid=S1665-80272016000100006

Roque, Y., Valdivia, P., Alonso, S., y Zagalaz, M. (2018). Metacognición y aprendizaje autónomo en la Educación Superior. Educación Médica Superior, 32(4), 293-302. http:// scielo.sld.cu/scielo.php?script=sci_arttext\&pid=So864-21412018000400024\&ln $\mathrm{g}=\mathrm{es} \& \mathrm{t} \operatorname{lng}=\mathrm{es}$.

Ruiz-Bernardo, P. (2018). Los espacios y la frecuencia de contacto con la diversidad cultural como factores de desarrollo de la sensibilidad intercultural. Aposta. Revista de Ciencias Sociales, (76), 187-213.http://repositori.uji.es/xmlui/ handle/10234/174740

Santillán, E. (2018). Nichos identitarios: aproximación metodológica al estudio de jóvenes indígenas, en M. Ayala. y G. García (Coords.). Diversidad metodológica en la investigación psicosocial (pp. 131-156). Mexicali: Universidad Autónoma de Baja California. Sociedad Mexicana de Psicología Social.

Schmelkes, S. (2021). Enseñar y aprender en contextos de exclusión. DIDAC, (77), 100-106. https://doi.org/10.48102/didac.2021..77\%20ENE-JUN.54

Soto, V. (2019). Colonialidad del poder y utopía del mercado total. Aportes de Quijano y Lander al pensamiento decolonial, Visioni LatinoAmericane è la rivista del Centro studi per l'America Latina, 11 (20), 58-72. DOI: 10.13137/2035-6633/22531

Velasco, S., y Baronnet, B. (2016). Racismo y escuela en México: Reconociendo la tragedia para intentar la salida. Diálogos sobre educación, (7), 1-17. https://doi. org/10.32870/dse.voi13.241

Walsh, C. (2017). Pedagogías decoloniales. Prácticas insurgentes de resistir, (re)existir, y (re)vivir. Tomo II. Quito:Abya-Yala. 